\title{
Oxigenoterapia hiperbárica no processo de cicatrização de feridas: revisão de literatura
}

\section{Hyperbaric oxygen therapy in the wound healing process: literature review}

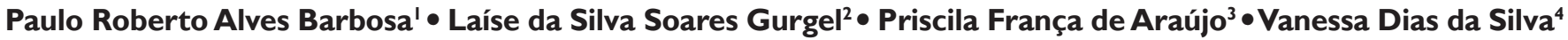

\begin{abstract}
RESUMO
Os objetivos deste estudo são conhecer as publicações científicas disponíveis nas plataformas on-line sobre o tratamento de feridas por meio da oxigenoterapia hiperbárica $(\mathrm{OHB})$ e compreender o papel da enfermagem na cicatrização de feridas com tratamento em OHB. Trata-se de uma revisão de literatura, realizada em outubro de 2019, nas bases de dados BVS Brasil Lilacs, Scielo, Medline e Pubmed, tendo como critérios de inclusão publicados a partir do ano de 1995, disponíveis na íntegra e gratuitos, realizados em seres humanos e nos idiomas português e/ou inglês.A seleção se deu por meio dos descritores Decs/ Mesh “Oxigenação hiperbárica/ Hyperbaric Oxygenation e enfermagem/ nursing”, utilizando o boleano "and”. Após leitura dos artigos, selecionaram-se quatro artigos para análise. Assim, emergiram as seguintes categorias temáticas: a fisiologia do tratamento, método e indicação do tratamento e atuação da equipe de enfermagem expressam uma necessidade emergente de publicações no que diz respeito à apropriação do campo da oxigenoterapia hiperbárica $(\mathrm{OHB})$ pela enfermagem, tendo em vista que, em II anos, após a inserção do enfermeiro na atuação do campo da OHB, foram encontrados poucos artigos direcionados ao profissional dessa área. Conclui-se que há necessidade de mais estudos, principalmente, os que possam descrever protocolos clínicos do uso da oxigenoterapia hiperbárica no intuito de prover suporte teórico-prático atualizado ao profissional em campo de atuação.

Palavras-chave: Oxigenação Hiperbárica. Ferimentos e Lesões. Enfermagem.
\end{abstract}

\begin{abstract}
The objectives of this study are to know the scientific publications available on the online plat-forms on the treatment of wounds through hyperbaric oxygen therapy $(\mathrm{HBO})$ and to under-stand the role of nursing in the healing of wounds treated with HBO.This is a literature re-view, carried out in October 2019, in the VHL Brazil Lilacs, Scielo, Medline and Pubmed databases, with the inclusion criteria: published since 1995, available in full and free of charge, carried out in human beings and in Portuguese and / or English languages. The selection was made through the descriptors Decs / Mesh "Hyperbaric oxygenation / Hyperbaric Oxygenation and nursing, using the Boolean "and". After reading the articles, 4 articles were selected for analysis. Thus, the following thematic categories emerged:The physiology of treatment, method and indication of treatment and performance of the nursing team. express an emerging need for publications regarding the appropriation of the field of hyperbaric oxygen therapy ( $\mathrm{HBO}$ ) by nursing, considering that in II years after the insertion of nurses in the field of $\mathrm{HBO}$, few articles were found directed to the professional of this area. It is concluded that there is a need for further studies, mainly those that can describe clinical protocols for the use of hyperbaric oxygen therapy in order to provide updated theoretical and practical support to the professional in the field.

Keywords: Hyperbaric Oxygenation. Injuries and Wounds. Nursing.
\end{abstract}




\section{INTRODUÇÃO}

A pele é o maior órgão do corpo humano, formada por uma dupla membrana que reveste todo o corpo, responsável por, aproximadamente, $16 \%$ do peso corporal. É um órgão funcional que reveste e protege o corpo humano, tendo como principal função a termorregulação e a capacidade de isolar o corpo de agressões externas ${ }^{(1)}$.

Por razões de rompimento desse tecido, ou seja, perda de sua integridade, origina-se o que clinicamente se chama de feridas. As feridas são classificadas por fatores que envolvem a causa, a origem microbiana, o tipo de cicatrização, o grau de abertura e o tempo de duração. Além disso, as feridas podem apresentar graves intercorrências, como as infecções que, se não tratadas corretamente, podem desencadear correções cirúrgicas como a amputação de um membro ${ }^{(2)}$.

É atribuição do profissional enfermeiro o tratamento de feridas, tais como a autonomia na decisão de coberturas, tanto no contexto hospitalar, como em unidades básicas de saúde, clínicas, consultórios e domicílios ${ }^{(3)}$.Além disso, é de sua responsabilidade o estabelecimento de uma política de avaliação dos riscos potenciais, por meio de escalas ou outras ferramentas validadas cientificamente, bem como a escolha de coberturas ${ }^{(3)}$.

Atualmente, são disponibilizadas, no mercado, diversas coberturas para o tratamento de lesões, como alginatos, hidrofibras, carvão ativado, papaína, filmes, pomadas e etc. Porém, ainda se observa que, dependendo do tipo ou estágio da lesão e dos fatores intrínsecos e extrínsecos do processo de cicatrização, há uma longa demanda de tempo para a cicatrização da lesão ${ }^{(4)}$.

Um dos tratamentos conhecidos atualmente para a diminuição do tempo de cicatrização é a câmara de oxigenoterapia hiperbárica (OHB), que surgiu em 1622, para fins medicinais, com o médico Henshaw, sendo expandida no século XIX, com Junod e Pravaz. Em 1965, são documentadas as primeiras aplicações de OHB em lesões cutâneas; pois, quando associadas ao ambiente de alta pressão, há um estímulo à vasoconstricção e elevação da proporção de oxigênio na corrente sanguínea, favorecendo, fisiologicamente, o processo de cicatrização da ferida ${ }^{(5)}$.

No Brasil, a OHB passa a ser considerada a partir de 1930; porém, restringia-se praticamente ao tratamento de casos de doença descompressiva (DD), que acometia mergulhadores. Em 1967, é fundada a Medicina do Mergulho, sendo estabelecido como o primeiro serviço de OHB, no Hospital Naval Marcílio Dias (HNMD), voltado para clientes de distintas indicações ${ }^{(6)}$.

Embora a existência desses registros, dados da Sociedade Brasileira de Medicina Hiperbárica (SBMH) consideram que a OHB chega ao Brasil em 1983 e somente em 1995 tem sua primeira Regulamentação de Medicina
Hiperbárica pelo Conselho Regional de Medicina do Estado de São Paulo e a Regulamentação Oficial da Medicina Hiperbárica pelo Conselho Federal de Medicina (CFM) em todo o Brasil pela Resolução 1.457/95(7).

A OHB configura-se como um tratamento capaz de ofertar oxigênio $(\mathrm{O} 2)$ a concentrações superiores a $21 \%$, sob o aumento de pressão atmosférica (ATA), com o objetivo de melhorar a hipoperfusão e a inflamação dos tecidos. Nessas condições, o O2 comporta-se como uma droga, provocando o aumento do metabolismo ${ }^{(8)}$.

O interesse pelo estudo surgiu durante vivência de estágio extracurricular em uma clínica que ofertava o serviço de OHB, na qual se observou que tal recurso não era amplamente divulgado no meio acadêmico. Assim, inquietou-se o desejo de difundir esse recurso, buscando minimizar o tempo de cicatrização das feridas, e, consequentemente, a melhora na qualidade de vida dos usuários.

Reconhecem-se os efeitos da OHB no processo de cicatrização, porém ainda existe uma baixa procura por esse serviço, podendo estar associada a pouca disseminação dos estudos entre profissionais da base operacional e dos usuários do serviço em geral. Nessa perspectiva, emergiu o seguinte questionamento: Quais publicações científicas abordam o papel do enfermeiro na assistência da oxigenoterapia hiperbárica no tratamento de feridas? Assim, objetivou-se conhecer as publicações científicas disponíveis nas plataformas on-line sobre o processo de cicatrização de feridas por meio da OHB e compreender - papel da enfermagem na cicatrização de feridas utilizando a OHB.

\section{MÉTODO}

Foi elaborada uma revisão de literatura, visto que essa é uma opção de estudo capaz de verificar o material disponível a fim de compreender e atualizar dados anteriormente publicados, fornecendo evidências integradas e imparciais nas quais as decisões se baseiam, identificando lacunas na literatura para estimular estudos futuros minimizando tendências ${ }^{(9)}$.

A coleta das informações deu-se durante o mês de outubro de 2019, realizando uma busca on-line a partir da Biblioteca Virtual em Saúde (BVS Brasil), Scientific Electronic Library Online (Scielo), Literatura Latino-Americana e do Caribe em Ciências da Saúde (LILACS), Sistema Online de Busca e Análise de Literatura Médica (Medline)Pubmed, empregando os descritores Decs/Mesh: "Oxigenação hiperbárica/ Hyperbaric Oxygenation "and" enfermagem/ nursing. Posteriormente, foram analisados os artigos que se encaixavam nos seguintes critérios de inclusão: publicados a partir do ano de 1995, ano no qual a OHB foi regulamentada no Brasil; disponíveis na íntegra e gratuitos, realizados em seres humanos, que abordas- 
sem sobre o tratamento de feridas com oxigenoterapia hiperbárica publicados em português e/ou inglês. Foram excluídos os artigos repetidos nas referidas bases eletrônicas e os que o título, o resumo e/ou os resultados não respondessem à pergunta norteadora.

A análise se deu mediante classificação do nível de evidência, em que são estabelecidos critérios que pontuam de I a 7, em que I representa um nível de evidência forte e 7 , nível de evidência fraco ${ }^{(10)}$.
Consideram-se nível I as evidências provenientes de revisão sistemática ou metanálise; nível 2 são as evidências derivadas de, pelo menos, um ensaio clínico randomizado controlado, bem delineado; no nível 3 , as evidências são obtidas de ensaios clínicos bem delineados sem randomização; no nível 4, as evidências são provenientes de estudos de coorte e de caso-controle bem delineados; no nível 5 , as evidências são originárias de revisão sistemática de estudos descritivos e qualitativos; no nível

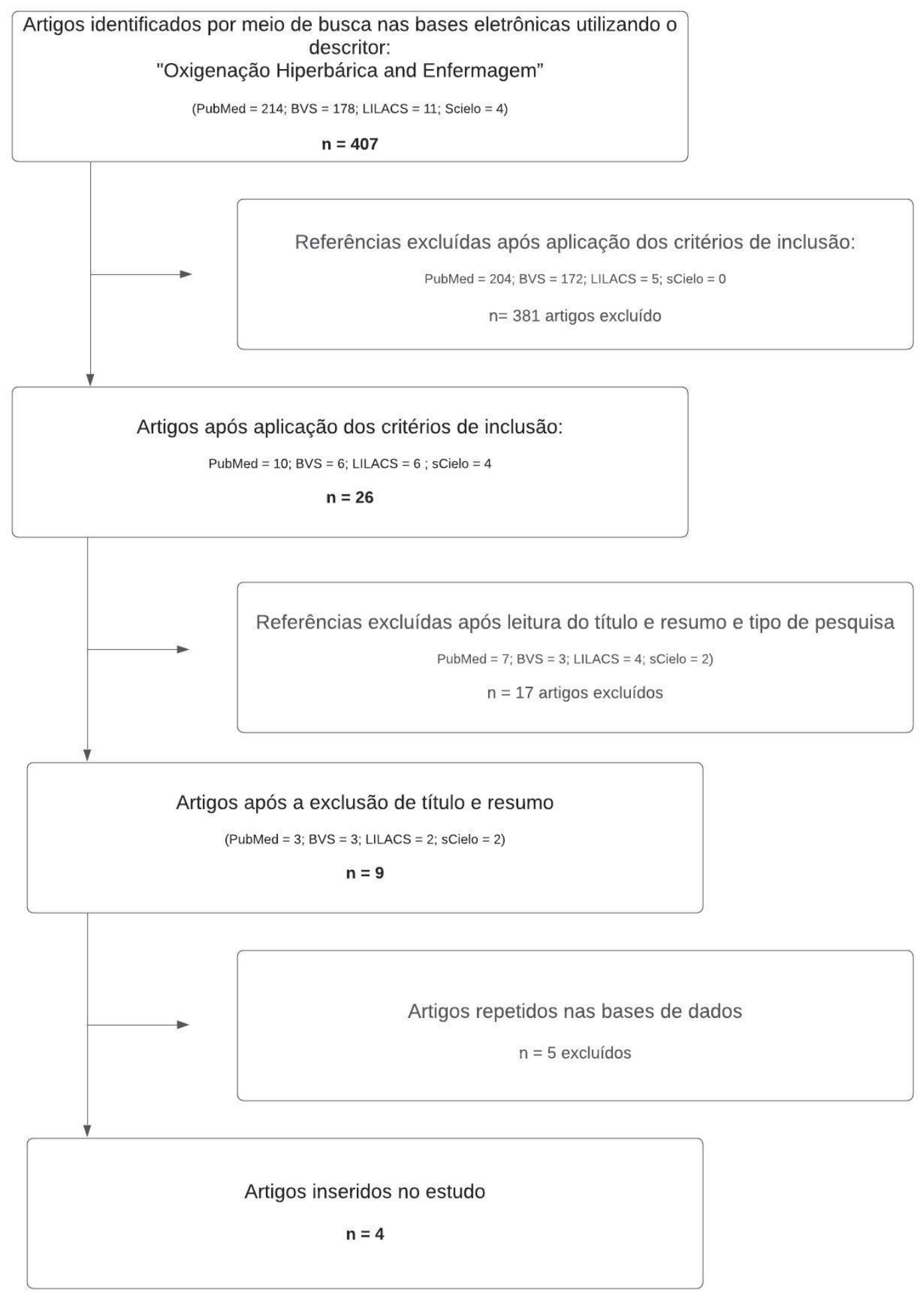

FIGURA 1 - Fluxograma da coleta e seleção dos dados, Fortaleza-CE, 2019.

Fonte: Autor da pesquisa, 2019. 
6, as evidências são derivadas de um único estudo descritivo ou qualitativo e, no nível 7 , as evidências são oriundas de opinião de autoridades e/ou relatório de comitês de especialistas ${ }^{(10)}$.

A apresentação e discussão dos resultados foram realizadas em dois eixos principais: I) caracterização dos estudos com base no instrumento de coleta de dados, classificação de grau de recomendação e nível de evidência científica; e 2) categorização com apresentação das principais contribuições das publicações em relação ao tema estudado, buscando delinear aspectos essenciais sobre a fisiologia do tratamento; o método e a indicação da OHB e a atuação do profissional enfermeiro.

\section{RESULTADOS}

Segundo a Sociedade Brasileira de Medicina Hiperbá- rica (SBMH), as diretrizes de segurança e qualidade determinam que, em 2003, as câmaras hiperbáricas comecem a ser operadas por técnicos em enfermagem, sendo, em 2008, incluídas pela SBMH e pelo capítulo brasileiro da Undersea Hyperbaric Medical Society (UHMS), a obrigatoriedade do enfermeiro como responsável técnico, assim como preconizado pelo Manual para Acreditação de Unidades Hiperbáricas Clínicas ${ }^{(11)}$.

Os artigos encontrados nas plataformas expressam uma necessidade emergente de publicações no que diz respeito à apropriação do campo da oxigenoterapia hiperbárica $(\mathrm{OHB})$ pela enfermagem, tendo em vista que, em II anos após a inserção do enfermeiro na atuação do campo da OHB, foram encontrados poucos artigos direcionados ao profissional dessa área, assim como exposto na tabela I.

TABELA 01 - Caracterização dos estudos conforme tipo de estudo, autor, objetivos, conclusão, ano de publicação e nível de evidência, Fortaleza- CE, 2019.

\begin{tabular}{|c|c|c|c|c|c|}
\hline $\mathbf{N}^{\circ}$ & Título/Tipo de estudo & Autor & Objetivo & Conclusão & $\begin{array}{l}\mathrm{NE}^{\star} / \text { Ano de } \\
\text { Publicação }\end{array}$ \\
\hline $1^{(12)}$ & $\begin{array}{l}\text { Arterial Disease Ulcers, } \\
\text { part 2: treatment } \\
\text { Estudo descritivo }\end{array}$ & Weir, et al. & $\begin{array}{l}\text { Desenvolver um quadro clínico } \\
\text { apropriado de abordagem } \\
\text { adequada às necessidades } \\
\text { individuais do paciente com } \\
\text { insuficiência arterial, baseado } \\
\text { no paradigma de preparação do } \\
\text { leito da ferida. }\end{array}$ & $\begin{array}{l}\text { O desenvolvimento de um } \\
\text { plano de ensino adequado } \\
\text { e individual ao tratamento } \\
\text { do paciente é essencial na } \\
\text { contribuição da qualidade } \\
\text { da assistência prestada } \\
\text { por uma equipe inter- } \\
\text { profissional. }\end{array}$ & $5 / 2014$ \\
\hline $2^{(13)}$ & $\begin{array}{l}\text { Atuação da enfermagem } \\
\text { no tratamento da } \\
\text { Oxigenoterapia } \\
\text { hiperbárica. } \\
\text { Relato de experiência }\end{array}$ & Lacerda, et al. & $\begin{array}{l}\text { Discorrer sobre a atuação de } \\
\text { enfermagem no tratamento de } \\
\text { Oxigenoterapia hiperbárica. }\end{array}$ & $\begin{array}{l}\text { A participação permanente } \\
\text { da enfermagem } \\
\text { hiperbárica, representa o } \\
\text { desenvolvimento de uma } \\
\text { nova pesquisa de mercado } \\
\text { de trabalho. }\end{array}$ & $6 / 2006$ \\
\hline $3^{(14)}$ & $\begin{array}{l}\text { Oxigenoterapia } \\
\text { hiperbárica para } \\
\text { tratamento de feridas. } \\
\text { Estudo transversal }\end{array}$ & Andrade, Santos & $\begin{array}{l}\text { Descrever os tipos mais } \\
\text { frequentes de feridas com } \\
\text { indicação para terapia por } \\
\text { oxigênio hiperbárico e os } \\
\text { resultados obtidos. }\end{array}$ & $\begin{array}{l}\text { A terapia por oxigênio } \\
\text { hiperbárico é um } \\
\text { tratamento efetivo para } \\
\text { pacientes com feridas } \\
\text { crônicas. }\end{array}$ & $6 / 2016$ \\
\hline $4^{(15)}$ & $\begin{array}{l}\text { Hyperbaric oxygen } \\
\text { therapy: Exploring the } \\
\text { clinical evidence. } \\
\text { Estudo descritivo }\end{array}$ & $\begin{array}{l}\text { Lam G, Fontaine } \\
\text { R; Ross FL, Chiu } \\
\text { ES }\end{array}$ & $\begin{array}{l}\text { Analisar a fisiologia e o } \\
\text { mecanismo de cicatrização } \\
\text { relacionado à Oxigenoterapia } \\
\text { hiperbárica. }\end{array}$ & $\begin{array}{l}\text { O mecanismo de ação } \\
\text { envolvido na cicatrização } \\
\text { de feridas crônicas por } \\
\text { Oxigenoterapia hiperbárica } \\
\text { inclui a promoção de } \\
\text { neovascularização e } \\
\text { diminuição da inflamação. }\end{array}$ & $7 / 2017$ \\
\hline
\end{tabular}


A escassez de publicações gratuitas disponíveis acerca de tal temática tem gerado viés em diversas pesquisas no campo de atuação hiperbárica, o que apoia a hipótese de que ainda é necessária maior apropriação do enfermeiro nesse campo de atuação, hipótese essa que é apoiada pelo artigo $3^{(14)}$ (tabela I) quando o autor conclui que a participação permanente da Enfermagem Hiperbárica representa o desenvolvimento de uma nova perspectiva de mercado de trabalho ${ }^{(14)}$.

Após seleção dos artigos e leitura aprofundada, emergiram as seguintes categorias temáticas: a fisiologia do tratamento, o método e a indicação do tratamento e a atuação da equipe de enfermagem, descritas a seguir.

\section{DISCUSSÕES}

\section{A fisiologia do tratamento}

A Oxigenoterapia hiperbárica (OHB) passa a existir em nosso País a partir de 1967, por meio da marinha do Brasil, no Hospital Naval Marcílio Dias (HNMD). Esse tratamento tem seus fundamentos nas leis da física de mergulho, ou seja, embasadas nas leis de Dalton, Henry e Boyle ${ }^{(6)}$.

$\mathrm{O}$ artigo $2^{(13)}$ foi o único a citar as leis de mergulho para justificar o mecanismo de estímulo ao processo de cicatrização; entretanto, é verificado, em todos os outros autores, o termo "neovascularização" que se trata do principal processo fisiológico para a cicatrização dessas feridas. Com esses níveis elevados, pode-se verificar o aumento da neovascularização, da matriz extracelular e a diminuição da inflamação (15).

Os artigos $2^{(13)}$ e $4^{(15)}$ se complementam no que se refere à fisiologia da $\mathrm{OHB}$, sendo possível compreender a fisiologia associada ao processo de cicatrização e, desse modo, refletir sobre o papel do enfermeiro na compreensão dos mecanismos de apoio para o entendimento do processo cicatricial, as bases científicas da cascata inflamatória e processo de cicatrização, possibilitando a compreensão do que os autores citam na formação de uma neovascularização como ganho primordial no que diz respeito ao tratamento com $\mathrm{OHB}$.

A neovascularização é provocada a partir do aumento de células como VEGF e TGF- $\square$, células relacionadas ao fator de crescimento vascular, o que provoca a formação de novos capilares. A compreensão desse fluxo neovascular é complementada pelo estudo de Lacerda, et al. (2006) $)^{(6)}$ que expõem que, durante as sessões de $\mathrm{OHB}$, após receberem altas taxas de oxigênio, os tecidos sofrem um processo de hipóxia pós OHB, o que acaba por estimular a neovascularização(15).

Sendo assim, fica compreendida a eficácia da OHB, tanto durante, como após o tratamento, visto que as altas taxas produzem matéria-prima necessária para a formação de novos vasos, assim como em baixos níveis, pós -estimulação exercida pelo ambiente hiperbárico, após o tratamento, em que há uma estimulação do corpo para prover suporte sanguíneo que resultará na neovascularização ${ }^{(6)}$.

Outro fator que contribui para esse processo é o aumento da matriz extracelular que provoca $\circ$ aumento de células do fator de crescimento de foroblastos (FGF), desencadeando uma proliferação e migração de fibroblastos e aumento na produção de colágeno. Esse processo acaba por proporcionar a chegada de concentrações adequadas de oxigênio em tecidos pouco vascularizados, favorecendo o processo de cicatrização ${ }^{(15,6)}$.

Sendo assim, compreende-se que a OHB não se trata de um método de curativo ou tratamento isolado, mas de um tratamento adjuvante que irá promover suporte necessário para cicatrização, proporcionando uma perfeita e coordenada cascata de eventos celulares, moleculares e bioquímicos, que resultem nas fases da cicatrização, sendo elas: fase inflamatória, fase de granulação e fase de remodelamento da ferida ${ }^{(16)}$.

Em suma, os processos de cicatrização, por vezes, são retardados por falta de suporte celular, molecular e bioquímico e por parte do indivíduo, com problemas fisiopatológicos como a diabetes, as doenças vasculares e etc. Portanto, o que a OHB visa ofertar é o suporte necessário para que o próprio corpo possa cumprir sua cascata de cicatrização, cabendo ao enfermeiro associar esse tratamento com coberturas que estimulem a formação tecidual necessária, e ao médico verificar a necessidade de antibioticoterapia necessária para profilaxia, prevenção ao tratamento de infecções relacionadas ao leito da ferida.

\section{MÉTODO E INDICAÇÃO DO TRATAMENTO}

Os artigos foram unânimes no que se refere ao apoio, ao incentivo, à discussão de métodos e indicações para se optar pelo tratamento em OHB. Entre as possibilidades de tratamento, existem dois possíveis equipamentos hiperbáricos, sendo denominados câmaras hiperbáricas monoplace / monopacientes ou multiplace/ multipacientes, que podem ser diferenciadas pela capacidade de comportar números diferentes de pacientes (15).

As câmaras monopacientes são equipamentos com compartimento para um único paciente o qual respira oxigênio puro comprimido. Nas câmaras multiplaces, vários pacientes são expostos ao ar pressurizado por meio de uma máscara facial, capa, ou um tubo endotraqueal. As vantagens das câmaras monopacientes é que elas oportunizam um tratamento individualizado com pressurização em pressão atmosférica (ATA) fielmente recomendada; enquanto as câmaras multipacientes fornecem uma mesma ATA para todos os pacientes; desse modo, 
recomenda-se que sejam tratados pacientes com o mesmo tipo de lesão por sessão ${ }^{(15)}$.

Quando falamos de ATA, partimos do panorama que, normalmente, estamos submetidos à pressão exercida pela atmosfera sobre nossos corpos, já que ela corresponde a I ATA. A cada 10 metros de profundidade, aumenta-se uma atmosfera à pressão sobre o corpo mergulhado, termo utilizado para determinar um corpo em pressão hiperbárica ${ }^{(6)}$.

Nos artigos selecionados, evidenciaram-se algumas divergências na profundidade terapêutica a ser adotada. As profundidades terapêuticas tratam, exatamente, da definição da ATA adequada para prover suporte terapêutico a determinado paciente; portanto, saber o nível de pressão a ser fornecida para o paciente é de fundamental importância tendo em vista os riscos associados à pressurização hiperbárica( ${ }^{(17)}$.

De modo geral, a OHB deve ser realizada com pressões acima de I,4 por se tratar do que se considera pressão acima da atmosférica.A pressão exata escolhida varia de acordo com o foco de tratamento, podendo variar mediante protocolo. $\mathrm{Na}$ prática clínica, as pressões aplicadas variam geralmente $2-3^{(17)}$.

Embora se adotem as medidas de 2-3 ATAs, utilizamse, usualmente, valores inferiores a esse, de modo a garantir uma maior continuidade do tratamento. No tratamento de feridas, os protocolos, geralmente, envolvem tratamentos de OHB de I,5 a 2 ATA por sessão de 20 a 40 tratamentos, podendo ser estendidos a um máximo de 60 tratamentos, com, no máximo, três sessões por dia, a depender do tratamento ${ }^{(15)}$.

Essas indicações são preconizadas por órgãos regulamentares como é o caso da UHSM e SBMH. As primeiras indicações foram determinadas pela UHSM em 1976, quando ainda eram nomeadas como Submarino Medical Society. Elas são publicadas bienalmente segundo evidências clínicas disponíveis ${ }^{(6-15)}$.

Para medida de confirmação, são expostas, na tabela, as principais indicações para OHB segundo a UHMS e SBMH:

Além das pressões hiperbáricas, as câmaras de oxigenoterapia, denominadas normobárica são geralmente utilizadas devido à riqueza de apoio científico, confirmando seu potencial de cicatrização e capacidade de prevenir a infecção da ferida e deiscência, particularmente, após a cirurgia ${ }^{(12)}$. Desse modo, o paciente deverá ser rastreado de acordo com a sua necessidade de introdução na terapêutica da $\mathrm{OHB}$, visto que, para esse tipo de tratamento adjuvante, deve-se triar a possibilidade de cura da lesão(12).

Assim, foram registrados tratamentos que englobam, em sua maioria, o tratamento de feridas crônicas, mas que não contraindicam o uso da OHB para lesões agudas. Estudo realizado em Salvador-Ba observou os seguintes tipos de feridas: 42 (2l\%) úlcera venosa, 42 (2I\%) lesão traumática, 34 (I7\%) pé diabético, 19 (9,5\%) úlcera arterial, I 8 (9\%) osteomielite, I $3(6,5 \%)$ lesões por radiação, 10 (5\%) deiscência cirúrgica, 8 (4\%) lesão por pressão, 4 (2\%) erisipela bolhosa, 3 (I,5\%) queimadura, 2 (I\%) fasceíte necrotizante, 2 ( $1 \%$ ) cisto pilonidal, 2 ( $1 \%$ lesões buco-maxilo faciais e I $(0,5 \%)$ piodermite gangrenosa ${ }^{(13)}$.

\section{Atuação da equipe de enfermagem}

A atuação do enfermeiro no processo de OHB destaca-se como uma área de atuação relativamente nova, cuja regulamentação data-se de 2008 pela SBMH, embora a enfermagem já houvesse atuado por meio dos técnicos em enfermagem desde 2003. Esse panorama tem ganha-

\section{Valores recomendados de ATA por tratamento}

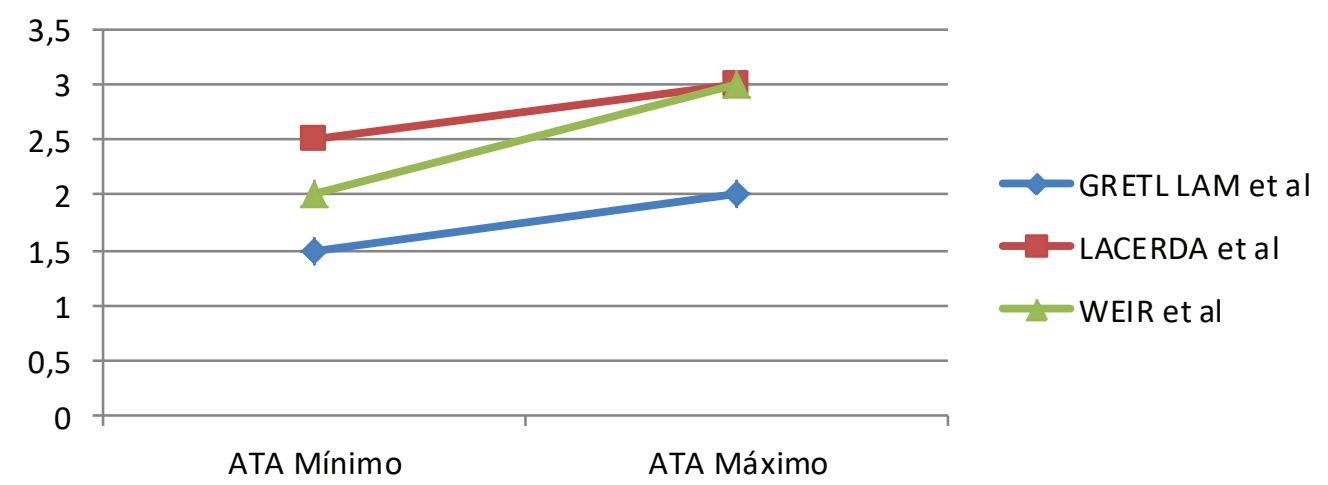

FIGURA 2 - Valores recomendados de ATA por tratamento, Fortaleza-CE, 2019.

Fonte: Autores da pesquisa, a partir dos artigos 1, 2 e 4. 
TABELA II - Protocolo de indicações para OHB, Fortaleza - CE, 2020.

\begin{tabular}{|c|c|c|}
\hline \multirow{2}{*}{ Indicação } & \multicolumn{2}{|c|}{ Protocolo } \\
\hline & ATA & $N^{0}$ de Sessões \\
\hline Intoxicação por monóxido de carbono & $2.5-3.0 \mathrm{QD}^{*}$ ou $\mathrm{BID}^{* *}$ & 5 \\
\hline Mionecrose clostridiana & $\begin{array}{l}3.0 \mathrm{TID}^{* * *}(1 \mathrm{dia}) \\
\mathrm{BID}^{* *}(4-5 \text { dias })\end{array}$ & 10 \\
\hline $\begin{array}{l}\text { Síndrome compartimental, esmagamento e } \\
\text { outras isquemias traumáticas }\end{array}$ & $\begin{array}{c}\mathrm{BID}^{* *}(2 \text { dias }) \\
\mathrm{QD}^{*} \text { (demais dias) }\end{array}$ & 9 \\
\hline Doença descompressiva & $2.8-6.0$ & 14 \\
\hline $\begin{array}{l}\text { Intensificação da cicatrização de feridas } \\
\text { problemáticas }\end{array}$ & $\begin{array}{c}2.0-2.5 Q^{*} \\
\text { ou BID** }\end{array}$ & 60 \\
\hline Infecções necrosantes de tecidos moles & $\begin{array}{c}2.0-2.5 \mathrm{BID}^{* *}(2 \text { dias }) \\
\mathrm{QD}^{*} \text { (demais dias) }\end{array}$ & 30 \\
\hline Osteomielite refratária & $2.0-2.5 Q^{*}$ & 30 \\
\hline Lesão tecidual por radiação & $2.0-2.4 Q^{*}$ & 60 \\
\hline $\begin{array}{l}\text { Enxertos e retalhos cutâneos } \\
\text { comprometidos }\end{array}$ & $2.0-2.5 \mathrm{QD}^{*}$ & $\begin{array}{l}20 \text { pré-cirurgia } \\
20 \text { pós-cirurgia }\end{array}$ \\
\hline Abscesso intracraniano & $2.5 \mathrm{QD}^{*}$ ou $\mathrm{BID}^{* *}$ & 12 \\
\hline Queimadura térmica & $\begin{array}{l}2.0 \mathrm{TID}^{\star * *} \text { (um dia) } \\
\mathrm{BID}^{\star *} \text { (demais dias) }\end{array}$ & 45 \\
\hline
\end{tabular}

Fonte: Lacerda, EP (2006)(6) . *QD= 1 sessão/dia; **BID=2 sessões/dia; ***TID=3 sessões/dia.

do, cada vez mais, destaque, de modo que, na atualização das especialidades em enfermagem, regulamentada pelo COFEN, em 2018, a enfermagem hiperbárica surge como uma área de especialização e não mais apenas como atu-

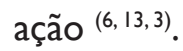

Uma pesquisa realizada com foco em úlceras arteriais relata que as terapias adjuvantes, como é o caso da OHB, podem melhorar a cicatrização desse tipo de ferida; no entanto, elas não corrigem a doença vascular subjacente, sugerindo-se uma avaliação multiprofissional para indicação de outras estratégias terapêuticas que colabore com o processo de cicatrização ${ }^{(12)}$.

Enfatiza-se que $\circ$ profissional de enfermagem hiperbárico deve ter experiência comprovada em atendimento a clientes críticos no que se refere à enfermagem médico-cirúrgica de adultos e crianças ${ }^{(19)}$. Porém, pouco se descreve sobre a real função do enfermeiro no campo de atuação, o que, à primeira vista, pode-se pensar que ela tenha sua atuação profissional mais focada na produção científica e pouca assistência prática.

Pode-se expressar como responsabilidade da enfermagem a relação de sua atuação em três momentos específicos do tratamento: o período pré-OHB, que consiste em medidas de conforto, relaxamento, proteção e conscientização do paciente no tocante à pressurização; ao período trans- OHB que consiste em suporte compensatório das cavidades aéreas nas mudanças de pressão, além do que, nas câmaras multipacientes, é necessária a presença de um profissional da equipe dentro da câmara para auxiliar na velocidade de pressurização de modo a manter o paciente o mais confortável possível para concluir o tratamento, e, por fim, o período pós -OHB que objetiva garantir a assistência completa ao paciente de OHB, permitindo-lhes a estabilidade geral para retornar ao seu local de origem ${ }^{(14)}$.

Além disso, cabe ao profissional enfermeiro manter constante acompanhamento da lesão, verificando se há ou não aceitação do paciente ao tratamento, análise da resposta da cascata inflamatória por meio da ferida, a fim de minimizar, ao máximo, a exposição do paciente aos ambientes hiperbáricos ${ }^{(6,13,19)}$.

\section{CONSIDERAÇÕES FINAIS}

Observou-se que há uma maior necessidade de estudos que possibilitem esclarecer e divulgar, cientificamente, a atuação do enfermeiro no tratamento adjuvante com OHBO, atuando como membro de uma equipe multidisciplinar que possibilita melhor assistência e evolução na cicatrização dessas lesões.

É notório que ainda existe a necessidade de fundamentar o campo da enfermagem hiperbárica acerca do seu processo de assistência, além da necessidade de estabelecer protocolos mais claros de atuação para esses profissionais, compreendendo o mecanismo de ação desse tratamento e apropriando-se desse campo de trabalho.

Em suma, este estudo se fundamenta como um suporte atualizado e sintetizado para a comunidade profissional acerca do tratamento, porém tem como fragilidade a escassez de artigos publicados sobre essa temática, o que nos possibilitou a análise de um número reduzido de artigos. 


\section{REFERÊNCIAS}

I. Junqueira LC, Carneiro J. Biologia celular e molecular. $9^{a}$ edição. Editora Guanabara Koogam. 2012.

2. Echer, IC. et al. Avaliação e tratamento de feridas: orientações ao profissionais de saúde [internet]. 20II [acesso em 20 out 2019] Hospital das clínicas. Porto Alegre. RS. Disponivel em: http://hdl.handle.net//0183/34755.

3. Cofen, Conselho Federal de Enfermagem. Resolução COFEN N $564 / 2017$ [internet]. 2017 [acesso em $29 \mathrm{abr}$ 2020] disponivel em: http://www.cofen.gov.br/resolucaocofen-no-56420I7_59I45.html

4 Silva AA, Schulz AVC, Costa AB. Caderno de enfermagem em ortopedia. Caderno de enfermagem. Volume 2. Fiocruz. Maio 2009. Rio de Janeiro.

5. Andrade MNB; Seward R; Melo JRC. Curativos. Rev Méd Minas Gerais [internet] 1992 [acesso em 05 set 2019] 2(4): 228-36 Disponível em: https://pesquisa.bvsalud.org/portal/ resource/pt/lil- 24547

6. Lacerda EP. Atuação da Enfermagem no Tratamento com Oxigenoterapia Hiperbárica. Rev Lat Amer Enferm [internet] 2006 [acesso em 21 out 2019] em: http://www.scielo. $\mathrm{br} / \mathrm{pdf} / \mathrm{rlae} / \mathrm{v}|4 \mathrm{nl} / \mathrm{vl} 4 \mathrm{nla}| 6 . p d f$

7. SBMH, Sociedade Brasileira de Medicina Hiperbárica. História da SBMH. [internet] 2002 [acesso em $05 \mathrm{dez}$ 2019] Disponivel em: https://sbmh.com.br/sobre/historia-da-sbmh/

8. Singhal AB. A review of oxygen therapy in ischemic stroke. Neurological Research:A Journal of Progress in Neurosurgery, Neurology and Neuro Sciences, 29(2), 173- I83. 2007.

9. Mendes S, Silveira P, Galvão M. Uso do gerenciador de referencias bibliográficas na seleção de estudos primários em revisão integrativa. Texto contexto - enferm. [internet]. 2019 [acesso em 04 nov 2019] Disponível em: http://www.scielo.hp?script=sci_arttext\&pi$\mathrm{d}=\mathrm{SO}$ I04-07072019000 I00602\&lng=en. Epub Feb 14,2019. http://dx.doi.org//0.1590//980-265x-tce-2017-0204

10. Melnyk BM, Fineout-Overholt E. Making the case for evidence-based practice. In: Melnyk BM, Fineout-Overholt E. Evidencebased practice in nursing \& healthcare. A guide to best practice. Philadelphia: Lippincot Williams \&
Wilkins;2005.p.3-24. [apud] Galvão CM. Níveis de Evidência [internet]. 2006 [acesso em 30 abr 2020] Rev.Acta Paul Enferm 19(2):V. Disponivel em: https://www.scielo.br/pdf/ ape/v19n2/a0Iv19n2.pdf

I I. SBMH, Sociedade Brasileira de Medicina Hiperbárica. Resolução do CFM da SBMH Nº 1457/95. [internet] 1995 [acesso em 05 dez 2019] Disponível em: https://sbmh.com.br/ sobre/resolucao-cfm/

12. Weir GR, Smart H, Van Marle J, Cronje FJ, Sibbald RG. Arterial Disease Ulcers, part 2: treatment. Clinical managment EXTRA. 2014 Oct;27(I0):462-76.

13. Lacerda EP,Alcantara LM, Leite JL, Trevizan MA, Costa Mendes IA, Uggeri CJR, Stipp MAC,Aspectos legais da enfermagem hiperbárica brasileira: porque regulamentar? Rev Bras Enferm, Brasília 2010 mar-abr; 63(2): 3I2-6.

14. Andrade SM; Santos ICRV. Oxigenoterapia hiperbárica para tratamento de feridas. Rev. Gaúcha Enfermagem [internet]. 2019 [acesso em 20 nov 2019]. vol.37, n.2, Disponível em: http://dx.doi.org/I0.1590/1983-1447.2016.02.59257.

15. Lam G, Fontaine R; Ross FL, Chiu ES. Hyperbaric oxygen therapy: Exploring the clinical evidence. Clinical managment EXTRA. 2017 Apr;30(4):18I-190.

16. Clark RAF. Wound repair. In: Kumar, Robbins, Cotran: Pathologic Basis of Disease, 7th ed. Ed. Saunders, p.I I2, 2005.

17. Howard MA, Asmis R, Evans KK, Mustoe TA. Oxigênio e ferida cuidados: uma revisão das modalidades terapêuticas atuais e futura direção.Wound Repair Regen 2013;21:503II. 4.

18. Leal TS, Oliveira BG, De Bomfim ES. Percepção de pessoas com a ferida crônica. Revenferm UFPE, Recife, I I (3): I I5662, mar., 2017.

19. Caixeta MAF. Manual de Oxigenoterapia Hiperbárica. Rio de Janeiro (RJ): Marinha do Brasil; 2003.

20. Brasil. Lei $n^{\circ}$ 10.74I. Estatuto do Idoso [internet] 2003 [acesso em 01 dez 2019] disponível em: http://www.planalto.gov.br/ccivil_03/LEIS/2003/LI0.74I.htm

Recebido: 2019-12-18 Aceito: 2020-07-09 